\title{
Prognostic value of tumoral and peritumoral magnetic resonance parameters in osteosarcoma patients for monitoring chemotherapy response
}

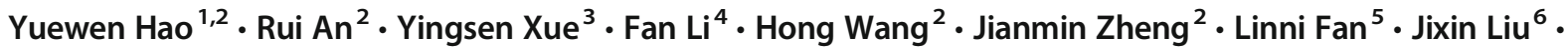 \\ Hongbin Fan ${ }^{7}$ Hong Yin ${ }^{2}$ (D)
}

Received: 11 February 2020 / Revised: 25 August 2020 / Accepted: 22 September 2020 / Published online: 4 November 2020

(C) The Author(s) 2020

\begin{abstract}
Objectives To evaluate parameters of diffusion-weighted imaging (DWI) and dynamic contrast-enhanced magnetic resonance imaging (DCE-MRI) as early imaging indicators of tumor histologic response to pre-operative neoadjuvant chemotherapy and as probable prognostic factors for event-free survival (EFS) and overall survival in osteosarcoma (OS) in both tumoral and peritumoral areas.

Methods Thirty-four OS patients who received three courses of neoadjuvant chemotherapy followed by surgery during 2014-2018 were enrolled in this study. All patients underwent baseline and post-chemotherapy DWI and DCE-MRI. Lesion region was defined as the tumoral area and peritumoral area. Parameters of apparent diffusion coefficient, capacity transfer constant (Ktrans), elimination rate constant, extravascular extracellular space volume ratio (Ve), and initial area under the curve as well as corresponding differences between pre- and post-chemotherapy in lesion regions were evaluated. Receiver operating characteristic analysis was used to evaluate the diagnostic performance of these parameters. The associations of all parameters with tumor histologic response, EFS, and overall survival were also calculated.

Results In the tumor area, moderate evidence was found that post-Ktrans was lower in responders as compared with that in poor responders ( $p=0.04$, false discovery rate [FDR] corrected), and $\Delta$ Ktrans exhibited significant between-groups differences $(p=$ 0.04, Bonferroni corrected; or $p=0.006$, FDR corrected). Weak evidence for the between-groups difference was found in the $\mathrm{Ve}$ in the peritumoral area $(p=0.025$ before treatment and $p=0.021$ after treatment, uncorrected). Furthermore, lower post-Ktrans in the tumoral area and lower pre-Ve in the peritumoral area were significant prognostic indicators for longer EFS $(p=0.002, p=$ 0.026 ) and overall survival ( $p=0.003, p=0.023)$.
\end{abstract}

Conclusions In OS, DWI and DCE-MRI parameters in both tumoral and peritumoral areas can reflect the chemotherapy response and prognosticate EFS and overall survival.

Yuewen Hao and Rui An contributed equally to this work.

Hongbin Fan

fanhb@fmmu.edu.cn

Hong Yin

yinhong@fmmu.edu.cn

1 Department of Radiology, Xi'an Children's Hospital, Xi'an, Shaanxi, China

2 Department of Radiology, Xijing Hospital, Fourth Military Medical University, Xi'an, Shaanxi, China

3 Department of Orthopaedic Surgery, Northwest Women's and Children's Hospital, Xi'an, Shaanxi, China
4 Department of Health Statistics, Fourth Military Medical University, Xi'an, Shaanxi, China

5 Department of Pathology, Fourth Military Medical University, Xi'an, Shaanxi, China

6 School of Life Science and Technology, Xidian University, Xi'an, Shaanxi, China

7 Department of Orthopaedic Surgery, Xijing Hospital, Fourth Military Medical University, Xi'an, Shaanxi, China 


\section{Key Points}

- Peritumoral MRI parameters can reflect the chemotherapy response in OS patients.

- Peritumoral MRI parameters can predict EFS and overall survival in OS patients.

- MRI parameters may be predictive factors for evaluating chemotherapy efficacy and EFS.

Keywords Osteosarcoma $\cdot$ Magnetic resonance imaging $\cdot$ Chemotherapy

$\begin{array}{ll}\text { Abbreviations } \\ \text { ADC } & \text { Apparent diffusion coefficient } \\ \text { AIF } & \text { Arterial input function } \\ \text { DCE } & \text { Dynamic contrast enhancement } \\ \text { DWI } & \text { Diffusion-weighted imaging } \\ \text { EFS } & \text { Event-free survival } \\ \text { IAUC } & \text { Initial area under the curve } \\ \text { Kep } & \text { Elimination rate constant } \\ \text { Ktrans } & \text { Capacity transfer constant } \\ \text { MRI } & \text { Magnetic resonance imaging } \\ \text { OS } & \text { Osteosarcoma } \\ \text { ROC } & \text { Receiver operating characteristic } \\ \text { ROI } & \text { Region of interest } \\ \text { TNR } & \text { Tumor necrosis rate } \\ \text { Ve } & \text { Extravascular extracellular space volume ratio } \\ \text { VIBE } & \text { Volumetric interpolated breath hold examination }\end{array}$

\section{Introduction}

Osteosarcoma (OS) is the most common malignant bone tumor with peak incidence in children and adolescents [1]. The current treatment strategy for OS is neoadjuvant chemotherapy that induces tumor necrosis to facilitate surgical resection. The combination of pre-operative and post-operative chemotherapy improved the long-term survival rate from 20 to nearly $70 \%$ in patients compared with surgery alone [2-6].

In OS, chemotherapy-induced necrosis is a well-accepted predictive factor for prognosis. Post-chemotherapy tumor necrosis in more than $90 \%$ cases is associated with significantly higher survival rate $[7,8]$. However, as necrosis can only be assessed after tumor resection post-chemotherapy, it may not represent a true early prognostic factor. Thus, it would be desirable to have a non-invasive method to predict clinical response to neoadjuvant chemotherapy before surgical resection.

Magnetic resonance imaging (MRI) has been developed to be the most important method for primary bone tumor diagnosis and post-operative tumor relapse detection. Among different MR measurements, dynamic contrast-enhanced MR imaging (DCE-MRI) (with its ability to measure tissue microvasculature properties such as tissue perfusion, capillary permeability, and interstitial volume $[9,10])$ and diffusionweighted (DW)-MRI (with its ability to assess impedance of water molecule diffusion, which is most dependent on the tissue cellularity [11-13]) have become the commonly used modalities in OS assessment. The change of capacity transfer constant (Ktrans) in DCE-MRI and apparent diffusion coefficient (ADC) in DW-MRI post-chemotherapy were correlated with pathological response in previous studies [14-20]. However, no parameter has been verified as a prognostic factor for patient outcomes in OS. Moreover, all previous studies focused only on the image parameter changes in the tumoral area, and the area adjacent to tumor was neglected. Slaughter et al suggested that the residual "alterated field" adjacent to the tumor could be the leading cause of treatment failure and local recurrence [21]. Several fundamental studies have demonstrated the association between tumor local recurrence and cancer-related gene expression in peritumoral area; however, whether clinical diagnostic imaging is correlated with tumor progression and treatment effect remains unclear, as it was hypothesized that parameters in peritumoral area detected by MRI would be possible indicators of response or prognostic factors for patient outcome of neoadjuvant chemotherapy.

In this study, we investigated the diagnostic and prognostic value of DW-MRI and DCE-MRI parameters in postchemotherapy OS patients in both tumoral and peritumoral areas.

\section{Materials and methods}

\section{Patients and treatment}

Thirty-four patients with high-grade non-metastatic and potentially resectable OS (median: 14 years, range 6-29 years; male $=18$, female $=16$ ) were enrolled in this study from January 2014 to June 2018 in the orthopedics department of Xijing Hospital, Fourth Military Medical University. Exclusion criteria were inability to give informed consent, history of surgical resection, radiotherapy or chemotherapy, non-lower extremity OS, and contraindications to MR or MR contrast media. Two patients deemed to be ineligible after enrollment were also excluded, resulting in a total of 34 patients in the study. This retrospective diagnostic study was approved by the institutional review committee of our center.

All patients received three courses of neoadjuvant chemotherapy before operation: ifosfamide $\left(12 \mathrm{~g} / \mathrm{m}^{2}\right)$ at week 9 , cisplatin $\left(60 \mathrm{~g} / \mathrm{m}^{2}\right)$ and doxorubicin $\left(120 \mathrm{mg} / \mathrm{m}^{2}\right)$ at week 6 , 
Fig. 1 Treatment schedule and timing of DW- and DCE-MR imaging. Base represents the baseline before treatment. I, ifosfamide; C, cisplatin; D, doxorubicin; W, week; $\mathrm{M}$, month
Chemotherapy

Postoperative

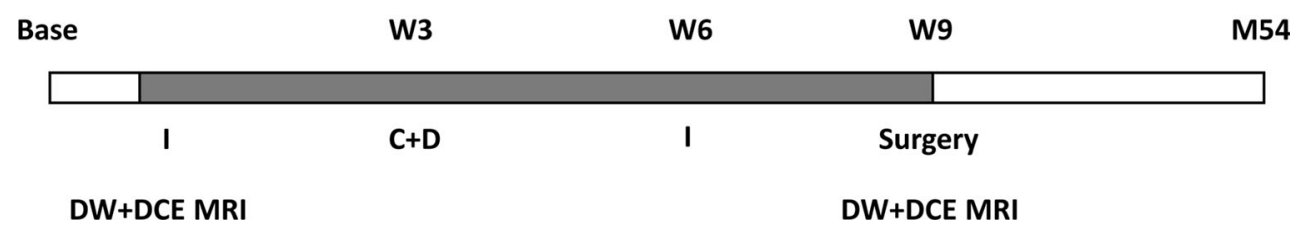

and ifosfamide $(12 \mathrm{~g} / \mathrm{m} 2)$ at week 3 before surgical resection. The treatment and imaging schedules are shown in Fig. 1.

\section{MRI data acquisition}

In this study, a 1.5-T MRI system (Siemens Healthineers) was used. All patients underwent MRI examinations twice including DCE-MRI and multi- $b$ value high-resolution diffusionweighted imaging examination at week 0 (before neoadjuvant therapy) and week 9 (before tumor resection/post-neoadjuvant therapy), respectively. A standard examination was performed including coronary and transverse fat-saturated $\mathrm{T}_{1} \mathrm{WI}$ and $\mathrm{T}_{2} \mathrm{WI}$.

For DCE-MRI protocols, firstly the T1 map was acquired by using the VFA method (variable flip angle technique) with the unenhanced T1-weighted VIBE (volumetric interpolated breath-hold examination) sequence, with flip angles of $2^{\circ}$ and $15^{\circ}$, which was automatically calculated by the Tissue 4D software (Siemens Healthineers) at the workstation. The patient was given injections of $0.1 \mathrm{mmol} / \mathrm{kg}$ of gadodiamide at a rate of $4 \mathrm{ml} / \mathrm{s}$ followed by a saline flush. Dynamic imaging was performed using the VIBE sequence with the parameters as follows: $\mathrm{TR} / \mathrm{TE}=3.79 / 1.35 \mathrm{~ms}$, slice thickness $=3.5 \mathrm{~mm}$, acquisition matrix $=340 \times 244$; the total acquisition time was almost $6 \mathrm{~min}$ for 60 measurements. Multi- $b$ value highresolution DWI examination was performed with the RESOLVE sequence (readout segmentation of long variable echo-trains), and the ADC value was generated automatically after scanning. The details of scan parameters are as follows: $\mathrm{TR}=2000 \mathrm{~ms}, \mathrm{TE} 1=59 \mathrm{~ms}, \mathrm{TE} 2=95 \mathrm{~ms}, 150^{\circ}$ flip angle, matrix $=360 \times 288$, slice thickness $=5 \mathrm{~mm}$, readout segments $=7$, echo spacing $=0.38 \mathrm{~ms}, b$ value $=0,50,100,150,200$, $300,500,800 \mathrm{~s} / \mathrm{mm}^{2}$; totally scan time was $6.16 \mathrm{~min}$.

\section{Evaluation of response and survival}

After definitive surgery at week 9, histologic response was assessed by experienced pathologists using the four-grade system of Huvos $[2,4]$. Tumor necrosis was graded as grade I ( 0 $49 \%)$, grade II (50-89\%), grade III (90-99\%), and grade IV $(100 \%)$. Patients with grades III and IV necrosis $(\geq 90 \%$ necrosis) are considered good responders (responder group) and those with grades I and II necrosis ( $<90 \%$ necrosis) as poor responders (poor-responder group).
For metastatic disease is an overwhelming factor in predicating survival, only patients with localized disease can be included in the analyses of event-free survival (EFS) and overall survival. EFS was defined as the time interval from the date of study enrollment to the date of the first event (disease progression, relapse, secondary malignancy, or death) or to the date of last follow-up for patients without events. Overall survival was defined as the time from the date of study enrollment to the date of death from any cause or to the last followup date.

\section{DCE-MRI analysis}

DCE-MRI raw dates were uploaded to the Siemens postprocessing station, and the Tissue 4D software was used to analyze DCE data. Tumor delineation was performed by two pediatric radiologists (clinical experience of more than 10 years) using an interactive display to select the region of interest (ROI) of the tumoral area on each slice that ensured that tumor boundary selection was consistent across all time points. The adjacent area of the ROI (peritumoral area/ molecular boundary) refers to the periphery area within $2 \mathrm{~cm}$ from the soft tissue mass (tumoral area). All measurements were kept away from the vessels to avoid interference of parameters.

Five tumoral areas were randomly delineated on the axis of ADC map and DCE map as ROI. The tumor region and peritumoral region of each layer were manually delineated, and the average ADC value was calculated.

Firstly, the motion correction of the images in each period was carried out; secondly, T1 mapping images were automatically registered; thirdly, by referring to the two-compartment pharmacokinetic model, AIF (arterial input function)-slow was fitted with TIC (time of intensity curve) type to generate false color images; fourthly, for each pixel inside the tumor region and peritumoral region ROI, the four quantitative parameters, Ktrans, elimination rate constant (Kep), extravascular extracellular space volume ratio (Ve), and IAUC, were computed.

The corresponding differences ( $\Delta$ means parameter postchemotherapy minus pre-chemotherapy, e.g., $\Delta \mathrm{K}$ trans $=$ postKtrans - pre-Ktrans) of each averaged kinetic parameter between pre- and post-neoadjuvant therapy of tumoral and peritumoral ROI were also computed for further statistical 
analysis. All parameters were used for assessing neoadjuvant therapy response, EFS, and overall survival.

\section{HE staining}

In this study, 34 patients underwent HE staining before and after chemotherapy. Paraffin sections were immersed in xylene for dewaxing for $15 \mathrm{~min}$, hematoxylin staining was performed for $2 \mathrm{~min}, 1 \%$ hydrochloric acid alcohol for $3 \mathrm{~min}$, water washing tablets, gradient alcohol for gradual dehydration, eosin staining for $2 \mathrm{~min}$, and neutral gum sealing piece.

\section{Statistical analysis}

Non-parametric Wilcoxon's signed-rank tests were used to examine the difference of ADC and eight DCE-MRI parameters (Ktrans, Kep, Ve, AUC, $\Delta$ Ktrans, $\Delta \mathrm{Kep}, \Delta \mathrm{Ve}$, and $\triangle \mathrm{AUC}$ in the ROI were determined for patients at two time points (week 0 [pre-chemotherapy] and week 9 [post-chemotherapy])). Mann-Whitney tests were used to examine the difference of each parameter between responders and poor responders. Bonferroni's and false discovery rate corrections were used for multiple comparison corrections. Receiver operating characteristic (ROC) curves were generated to compare parameters for differentiating responders and poor responders. Area under the curve, standard error, 95\% confidence interval (95\% CI), and optimal cutoff were estimated in the ROC analyses.

Univariate and multivariate analyses of EFS/overall survival at different areas (tumor or peritumoral area) were performed utilizing Cox proportional hazard model to explore associations between outcomes (EFS and overall survival) with each parameter, to adjust for potential confounding variables. Only covariates that were associated with the risk of

Table 1 Patient characteristics

\begin{tabular}{ll}
\hline Variable & Number of patients $(\mathrm{n}=34)$ \\
\hline Gender (male: female) & $18: 16$ \\
Age & Years \\
Median age (range) & $14(6-29)$ \\
Mean age \pm SD & $15 \pm 5.4$ \\
Primary sites & Number of patients $(\%)$ \\
Upper end of tibia & $14(41 \%)$ \\
Lower end of femur & $20(59 \%)$ \\
Pathological grade & III-IV \\
Metastasis & $0(0)$ \\
Tumor necrosis rate & \\
TNR $>90 \%$ & 19 \\
TNR < $90 \%$ & 15 \\
\hline
\end{tabular}

$S D$ standard deviation primary study endpoint at univariate analysis with $p<0.10$ were then included in the multivariate analysis. Selection of variables included in the multivariate model was done with backward elimination based on covariates that were significantly associated with the primary efficacy endpoint $(p>0.10$ for exclusion). Then, patients were categorized into two groups using the significant median parameter values as cutpoints. EFS and overall survival distributions were estimated using the method of Kaplan and Meier, and differences were examined using the log-rank test.

All the statistical analyses were performed using the Prism 6.02 and SAS software (version 9.1); two side $p$ values $<0.05$ were considered statistically significant.

\section{Results}

A total of 34 patients were available for analysis in the study. The responder group consisted of 19 patients (55.9\%) and the poor-responder group of 15 patients $(44.1 \%)$ (shown in Table 1). All 34 patients underwent surgery; in 26 patients, limb salvage surgery was performed while the other 8 patients underwent amputation. ROI of tumoral and peritumoral areas were delineated by radiologists with computer software as shown in Fig. 2a. All results for tumoral and peritumoral areas were compared.

\section{Imaging and histologic response}

First, we compared the parameters of pre- (week 0) and postneoadjuvant therapy (week 9) between groups (Table 2). In the tumoral area, post-Ktrans of the responder group was lower than that of the poor-responder group ( $p=0.04$, FDR corrected), and $\Delta$ Ktrans exhibited a significant betweengroups difference $(p=0.04$, Bonferroni corrected; or $p=$ 0.006 , FDR corrected). In the peritumoral area, post-ADC of the responder group was higher than that of the poorresponder group ( $p=0.01$, FDR corrected), and $\triangle \mathrm{ADC}$ exhibited a significant between-groups difference $(p=0.008$, FDR corrected). Additionally, weak evidence for the between-groups differences was also found in the Ve at different time points ( $p<0.05$, uncorrected).

Second, we measured the pre- and post-chemotherapy parameters in two groups with the self-contrasted method (Table 3). In the responder group, Ktrans and ADC exhibited significant changes both in the tumoral area and in the peritumoral area $(p<0.05$, corrected). In the poorresponder group, only ADC showed significant changes both in the tumoral area and in the peritumoral area $(p<$ 0.05 , corrected). 
Fig. 2 a The contrast-enhanced image, DCE-MR map (Ktrans as example), and ADC map in baseline examination (upper row) and post-chemotherapy (lower row) are displayed from left to right. The green line is the boundary of tumor ROI, and the yellow line is the boundary of peritumoral ROI drawn by a radiologist. The color bar is displayed on the left. $\mathbf{b}$ Picture 1, morphological observation in the tumor area before chemotherapy by HE staining (original magnification: $\times 100$ ). Picture 2, morphological observation in the tumor area after chemotherapy. Picture 3, morphological observation in the peritumoral area before chemotherapy. Picture 4, morphological observation in the peritumoral area after chemotherapy.

a

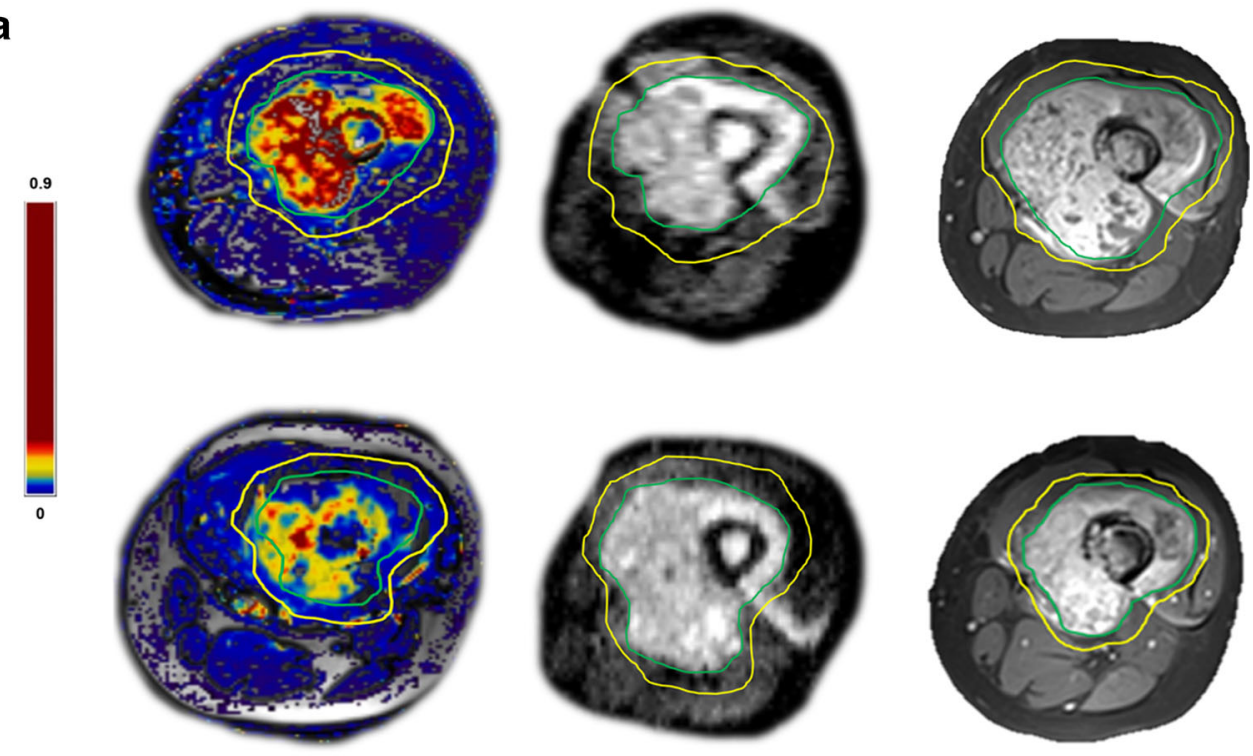

b

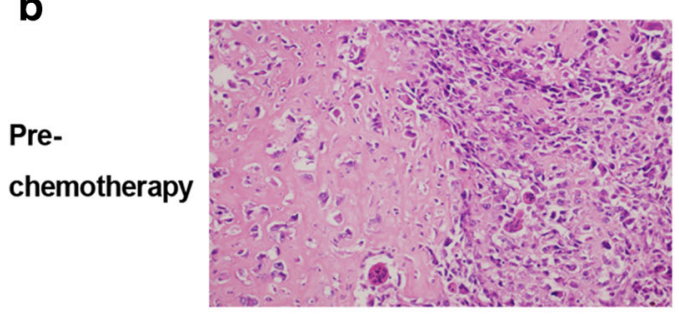

Tumor area

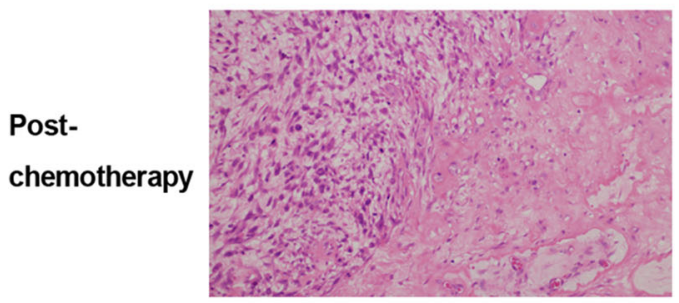

Tumor area

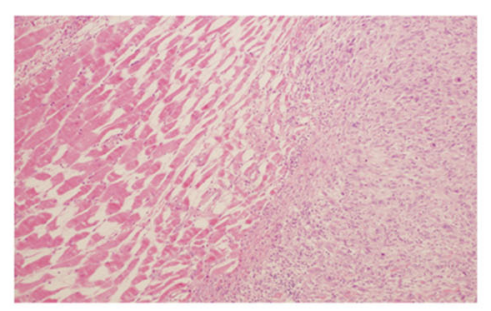

Peritumoral area

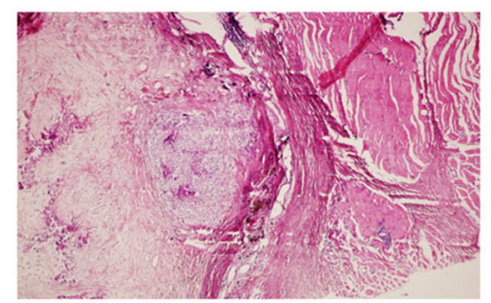

Peritumoral area

\section{HE staining of tumoral and peritumoral areas}

The results of the tumoral area showed that the heteromorphic cells were oval or short fusiform; epithelioid and transparent cells were arranged in sheets and nests, with ribbon-like bone matrix, hyperchromatic nuclei, mitotic figure, partial bone trabecular degeneration and calcification, and proliferation of vascular fiber tissue. Gross pathological specimens were taken after the surgery and stained with HE after chemotherapy, large areas of necrotic cells were observed under the microscope, and few tumor cells survived. The results of HE staining showed that normal muscle tissue could be seen outside the tumor cells before chemotherapy. After chemotherapy, large areas of necrotic cells were observed under the microscope in the tumoral area, and few tumor cells survived, with a little calcification and pigmentation. A small number of muscle cells were seen in the para-tumoral area, and no obvious signs of tumor cell infiltration were observed.

\section{Comparison of ROC curves}

We generated best threshold values for above-mentioned significant different MRI parameters as determined by the ROC curves. The sensitivity and specificity of the respective parameters were also measured at each threshold value to predict good histological response. 


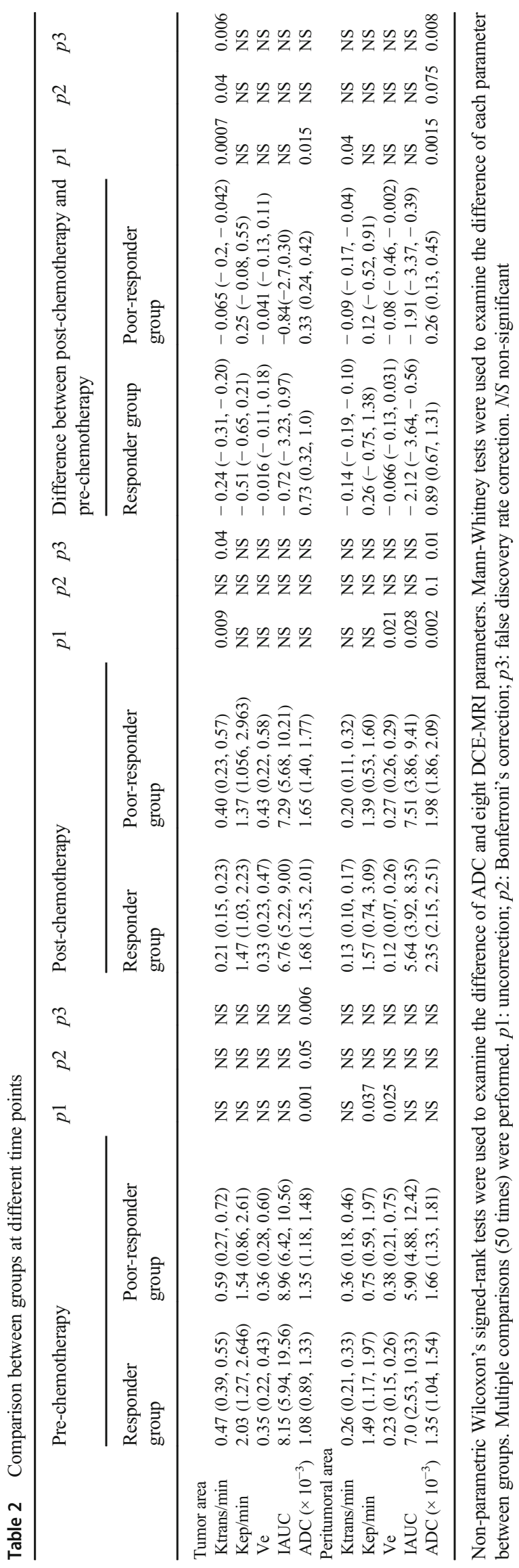


Table 3 Comparison between different time points

\begin{tabular}{|c|c|c|c|c|c|c|c|c|c|c|}
\hline & \multicolumn{2}{|l|}{ Responder group } & \multirow[t]{2}{*}{$p 1$} & \multirow[t]{2}{*}{$p 2$} & \multirow[t]{2}{*}{$p 3$} & \multicolumn{2}{|l|}{ Poor-responder group } & \multirow[t]{2}{*}{$p 1$} & \multirow[t]{2}{*}{$p 2$} & \multirow[t]{2}{*}{$p 3$} \\
\hline & $\begin{array}{l}\text { Pre- } \\
\text { chemotherapy }\end{array}$ & $\begin{array}{l}\text { Post- } \\
\text { chemotherapy }\end{array}$ & & & & Pre-chemotherapy & Post-chemotherapy & & & \\
\hline \multicolumn{11}{|l|}{ Tumor area } \\
\hline Ktrans/min & $0.47(0.39,0.55)$ & $0.21(0.15,0.23)$ & 0.0006 & 0.03 & 0.006 & $0.592(0.267,0.718)$ & $0.395(0.228,0.567)$ & NS & NS & NS \\
\hline $\mathrm{Kep} / \mathrm{min}$ & $2.03(1.27,2.64)$ & $1.46(1.03,2.23)$ & NS & NS & NS & $1.544(0.856,2.610)$ & $1.371(1.056,2.963)$ & NS & NS & NS \\
\hline $\mathrm{Ve}$ & $0.35(0.22,0.43)$ & $0.33(0.23,0.47)$ & NS & NS & NS & $0.360(0.278,0.602)$ & $0.428(0.222,0.582)$ & NS & NS & NS \\
\hline IAUC & $8.15(5.94,19.6)$ & $6.76(5.22,8.99)$ & NS & NS & NS & $\begin{array}{c}8.962(6.422 \\
10.560)\end{array}$ & $\begin{array}{c}7.293(5.684 \\
10.210)\end{array}$ & NS & NS & NS \\
\hline $\mathrm{ADC}\left(\times 10^{-3}\right)$ & $1.08(0.89,1.33)$ & $1.68(1.35,2.01)$ & 0.000002 & 0.0001 & 0.0001 & $1.350(1.180,1.480)$ & $1.650(1.400,1.770)$ & 0.0002 & 0.001 & 0.003 \\
\hline \multicolumn{11}{|l|}{ Peritumoral area } \\
\hline Ktrans/min & $0.26(0.21,0.33)$ & $0.13(0.10,0.17)$ & 0.0001 & 0.006 & 0.002 & $0.362(0.178,0.464)$ & $0.199(0.109,0.316)$ & NS & NS & NS \\
\hline $\mathrm{Kep} / \mathrm{min}$ & $1.49(1.17,1.97)$ & $1.57(0.74,3.09)$ & NS & NS & NS & $0.753(0.587,1.969)$ & $1.391(0.534,1.596)$ & NS & NS & NS \\
\hline $\mathrm{Ve}$ & $0.23(0.15,0.26)$ & $0.12(0.07,0.26)$ & NS & NS & NS & $0.377(0.206,0.751)$ & $0.269(0.257,0.291)$ & NS & NS & NS \\
\hline IAUC & $\begin{array}{c}7.01(2.53 \\
10.33)\end{array}$ & $5.64(3.92,8.35)$ & NS & NS & NS & $\begin{array}{c}5.945(4.882 \\
12.420)\end{array}$ & $7.511(3.857,9.406)$ & NS & NS & NS \\
\hline $\mathrm{ADC}\left(\times 10^{-3}\right)$ & $1.35(1.04,1.54)$ & $2.35(2.15,2.51)$ & 0.00004 & 0.002 & 0.001 & $1.655(1.325,1.810)$ & $1.980(1.858,2.085)$ & 0.0008 & 0.04 & 0.00 \\
\hline
\end{tabular}

Non-parametric Wilcoxon's signed-rank tests were used to examine the difference of ADC and eight DCE-MRI parameters. Mann-Whitney tests were used to examine the difference of each parameter between different time points. Multiple comparisons (50 times) were performed. $p 1$ : uncorrection; $p 2$ : Bonferroni's correction; $p 3$ : false discovery rate correction. $N S$ non-significant

\section{Imaging and event-free and overall survival}

A Cox proportional hazards model was used to analyze the associations between all MR parameters and EFS/overall survival. As shown in Fig. 3a, in the tumoral area, lower post-Ktrans was significantly associated with longer EFS $(p<0.01)$. In peritumoral area, lower pre-Ve (pre-neoadjuvant therapy/week 0) was significantly associated with longer EFS $(p=0.03)$. Moreover, analysis of the average post-Ktrans in the tumoral area as well as pre-Ve in the peritumoral area was performed using Kaplan-Meier curves stratified according to the median values for the cohort to explore the prognostic effect of these parameters. As shown in Fig. 4, patients with lower values of tumor post-Ktrans had a significant higher EFS rate $(p<0.001)$. Patients with lower values of peritumoral pre-Ve had a significant higher EFS rate $(p=0.017)$.

Overall survival was also analyzed with the same method. As shown in Fig. 5, in the tumoral area, lower pre- and post-Ktrans were significantly associated with longer OS $(p=0.023, p<0.001)$. In the peritumoral area, lower pre- and post-Ve were significantly associated with longer OS $(p=0.014, p=0.025)$, and higher postADC was significantly associated with longer OS $(p=0.036)$.

\section{Discussion}

In this study, DW- and DCE-MRI parameters in both tumoral and peritumoral areas were analyzed to explore their relationships with neoadjuvant therapy outcomes in OS patients. First, Ktrans was significantly decreased and ADC was significantly increased post-chemotherapy in responders and poor responders in both areas. Second, $\Delta$ Ktrans and $\triangle \mathrm{ADC}$ were significantly different between groups in both the areas and well correlated with histologic response. Third, post-Ktrans in the tumoral area and pre-Ve in the peritumoral area were significant prognostic factors for both EFS and overall survival.

OS, with peak incidence in children and adolescents, is the most common primary malignancy of bone. The main cause of mortality in patients with OS is lung metastasis, and approximately $10 \sim 20 \%$ of patients are diagnosed with metastatic OS upon identification of the disease in the USA [22]. Prognosis for OS patients has greatly improved since the introduction of effective chemotherapeutic agents in the 1970s and subsequent developments in neoadjuvant chemotherapy. The chemotherapy regimen that was adopted in this study was mentioned previously as most frequently used in our orthopedics department.

The treatment response of OS is commonly verified by the necrosis rate with pathological examination after tumor resection which is the last procedure in the treatment process; thus, imaging diagnosis such as MR imaging was urgently needed to replace the lagged indicator for early evaluation response. DW and DCE-MRI were the most frequently used in tumor diagnosing with their own typical features, reflecting tumor characteristics. DW-MRI well reflected the membrane 
A

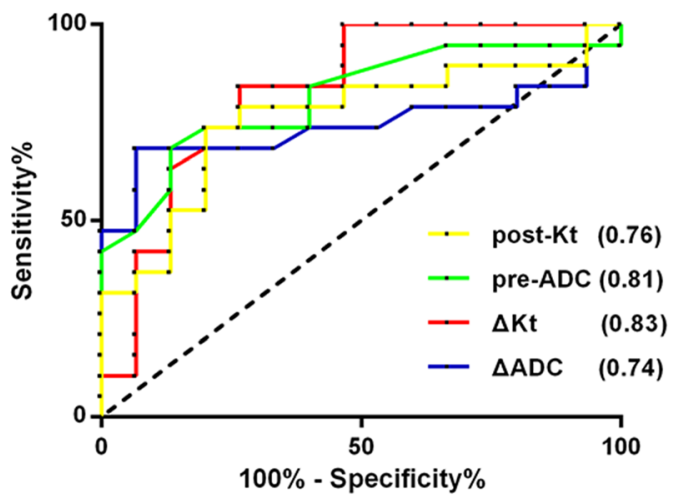

B

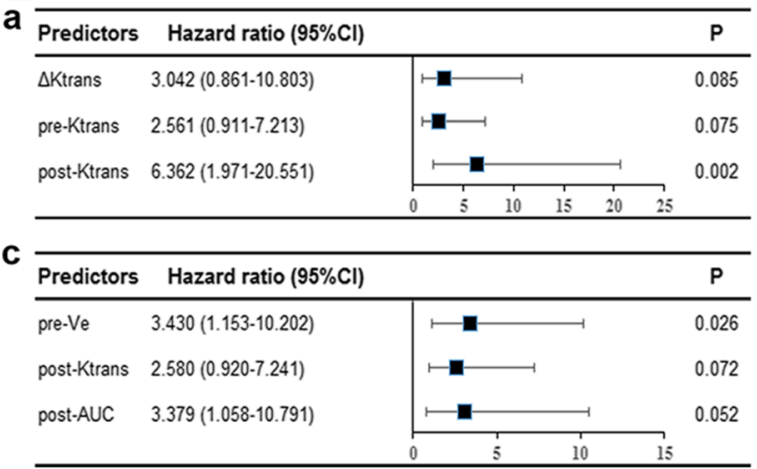

b
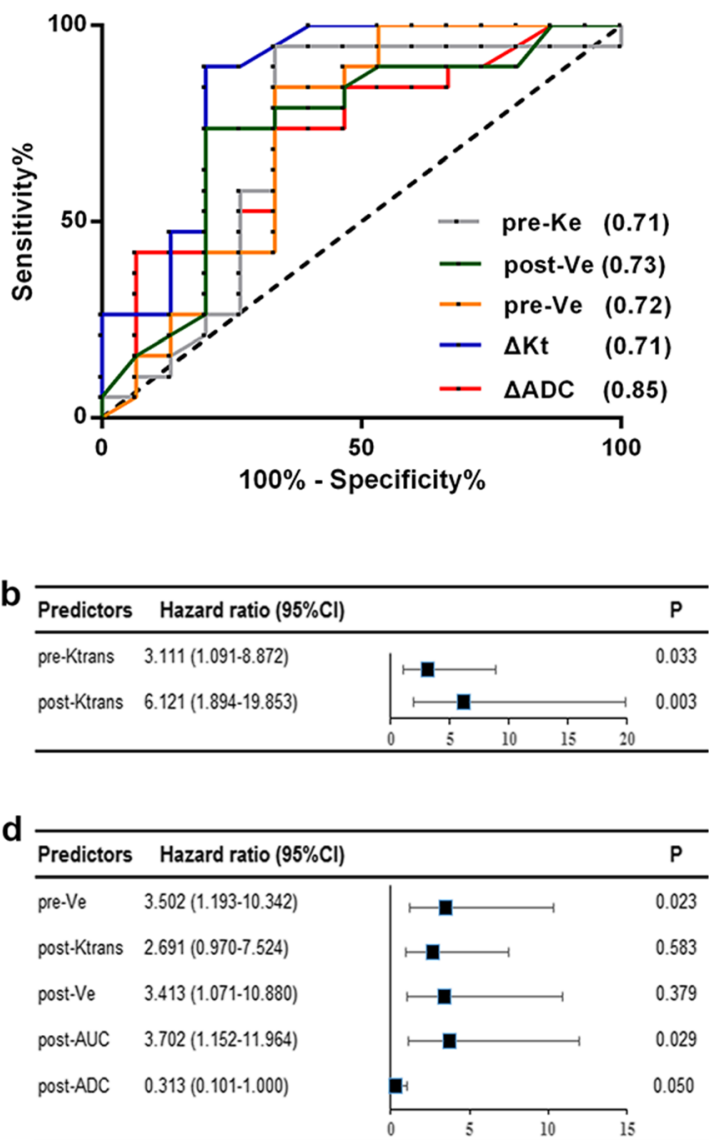

Fig. 3 A ROC curve for parameters in tumor and peritumoral areas. (a) tumor area; (b) peritumoral area. B Cox hazard models were used to estimate the hazard ratios and $95 \%$ confidence intervals of DW- and DCE-MRI parameters in association with EFS/overall survival. CI, confidence interval

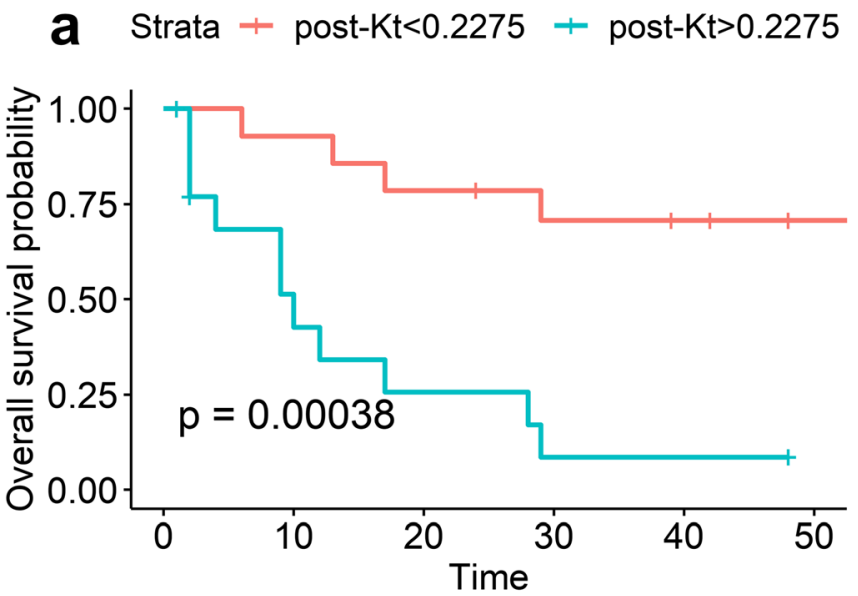

b Strata + pre-Ve $<0.2485+$ pre-Ve $>0.2485$

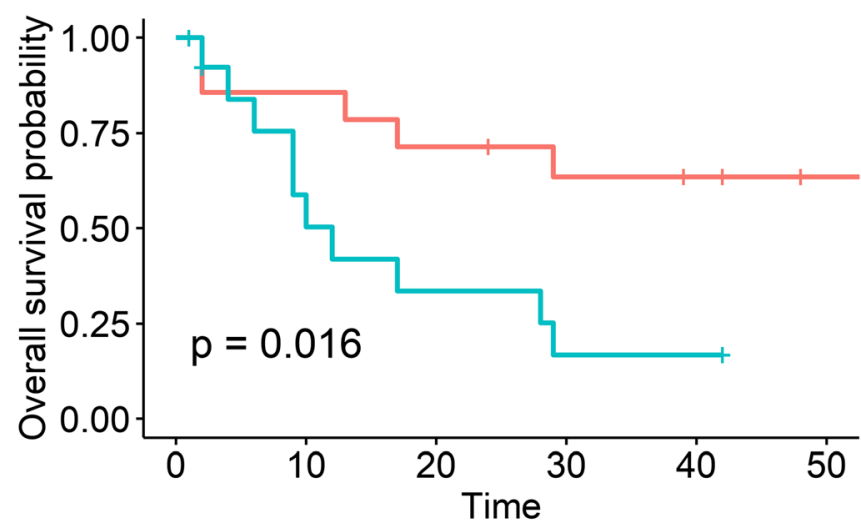

Number at risk

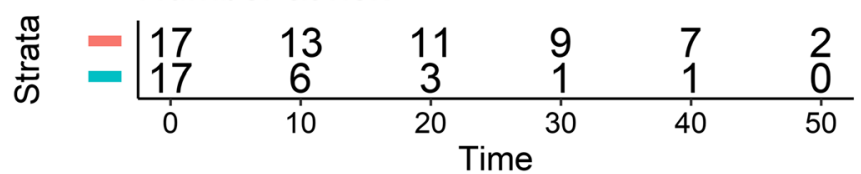

Number at risk

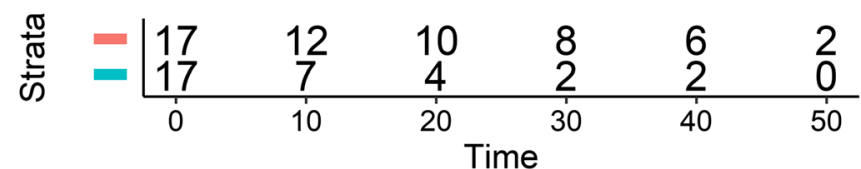

Fig. 4 Event-free survival curves for subgroups stratified by the median value of post-Kt in the tumor area (a) and pre-Ve in the peritumoral area (b). $p$ values were obtained from exact log-rank tests 
a Strata + pre-Kt $<0.4945+$ pre-Kt $>0.4945$

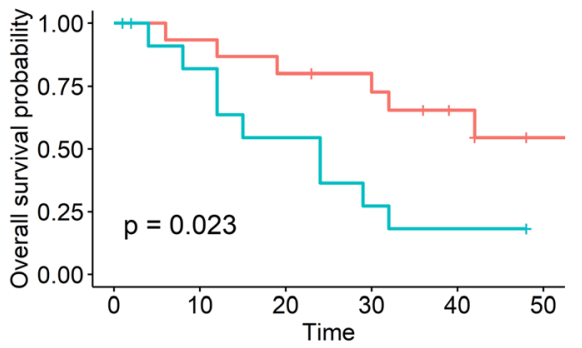

b

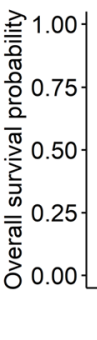

Strata + pre-Ve $<0.2485+$ pre-Ve $>0.2485$

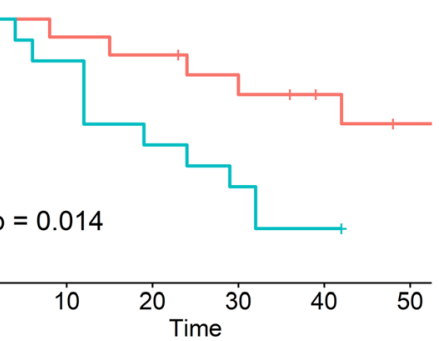

C Strata + post-ADC $<0.256+$ post-ADC $>0.256$

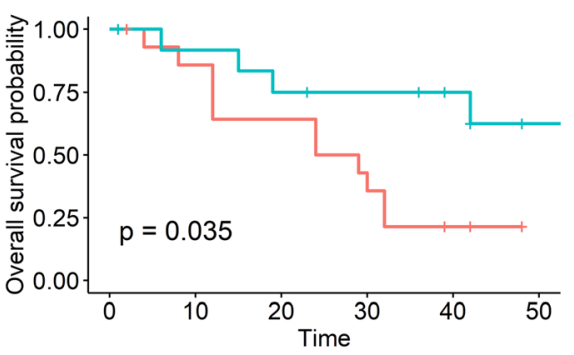

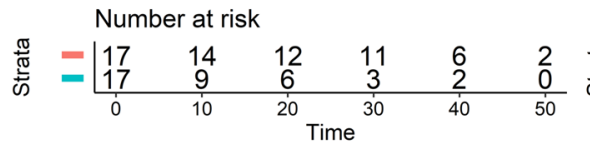

Number at risk
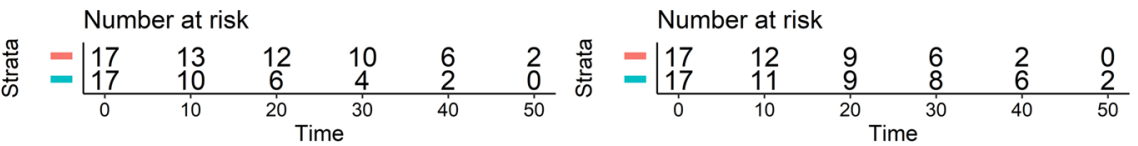

d Strata + post-Kt $<0.2275+$ post-Kt $>0.2275$

e Strata + post-Ve $<0.256+$ post-Ve $>0.256$
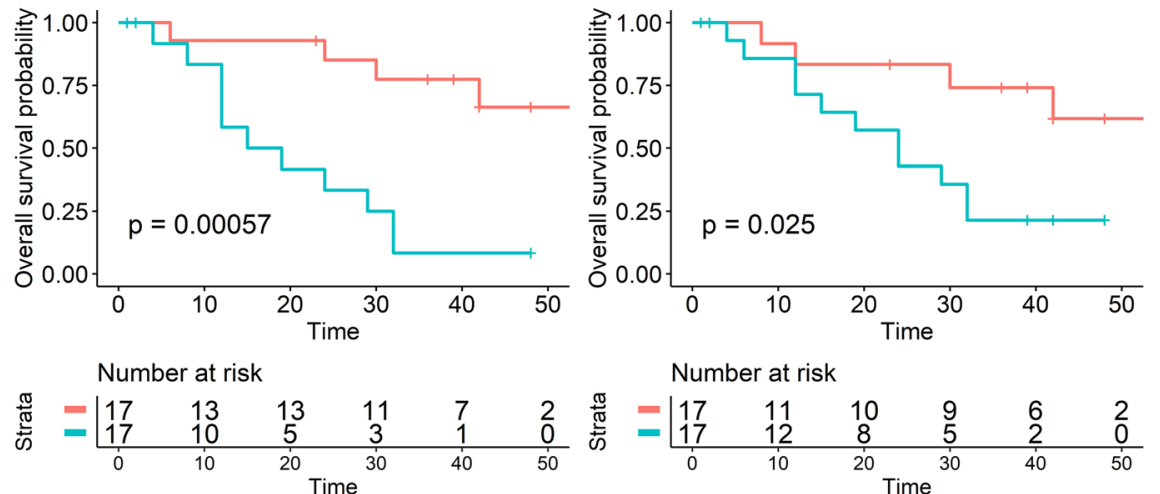

Fig. 5 Overall survival curves for subgroups stratified by the median value of pre-Kt (a) and post-Kt (b) in the tumor area; stratified by the median value of pre-Ve (c), post-Ve (d), and post-ADC (e) in the peritumoral area. $p$ values were obtained from exact log-rank tests

permeability between intra- and extracellular compartments with the surrogate marker of water diffusion, and DCE-MRI better indicated the leaky newly formed tumor capillaries, reflected by the contrast medium uptake capacity. Several studies have reported that ADC changes are greater in osteogenic patients with higher necrosis rate $(>90 \%)$ than in those with low necrosis rate $(<90 \%)$ post-chemotherapy [23], and lower post-chemotherapy Ktrans was associated with greater necrosis percent and longer event-free survival in OS patients [24]. Consistent with other studies, all patients in week 9 (post-chemotherapy) had higher ADC and lower Ktrans values than in week 0 (pre-chemotherapy), which means tumors were directly affected by chemotherapy drugs. Subtle changes in the degree of restriction of diffusion such as an alteration in cell membrane integrity or permeability to water, are reflected in changes in the DW signal [25]. In our study, in the tumoral area, $\triangle \mathrm{ADC}$ in the responder group was significantly higher than in the poorresponder group, and the higher necrosis rate reflected lower restriction of water molecule motion. $\Delta$ Ktrans in the responder group was also significantly higher than that in the poor-responder group; similarly, larger necrosis indicated decreased capillary permeability and reflected better response. Correspondingly, parameters in the peritumoral area had similar $\Delta \mathrm{K}$ trans and $\triangle \mathrm{ADC}$ variation trend. ROC curves indicated that among all parameters in both tumor and peritumoral areas that reflected histologic response, $\Delta \mathrm{K}$ trans in the tumoral area and $\Delta \mathrm{ADC}$ in the peritumoral area had the best diagnostic values.

MRI parameters can potentially reflect early changes in treatment especially in patients who resist or are unable to receive surgery for histologic response assessment. Currently, the existence of metastasis or not is the key prognostic factor that determines the treatment protocols for OS, and there is a pressing need to identify prognostic factors especially for the majority of patients who have localized disease. The prognostic significance of the DW- and DCE-MRI parameters will be useful to stratify patients in clinical trials and to identify the group of patients with private customized protocols.

In this study, it was found that no tumor cells were observed in the peritumoral area before and after chemotherapy by HE staining, but the targeted genes or specific molecules in the edema zone were over-expressed to varying degrees, and their biological behaviors were also changed. We hypothesized that peritumoral capillary permeability would change; in fact, the research finding showed that DCE-MRI parameters had changed before and after neoadjuvant chemotherapy, which was the imaging basis of DCE-MRI. 
The DCE-MRI parameters have been shown to be associated with clinical outcome manifested as histologic response and survival in some trials. Reddick et al found that lower Kep postneoadjuvant therapy was significantly predictive of EFS [26]. This is very similar to our results in which a lower post-Ktrans in the tumoral area was significantly associated with better EFS. Several studies have demonstrated that Ktrans was positively correlated with the tumors' malignancy grade, where neoformed leaky tumor capillaries can determine a rapid gadolinium uptake and an early wash-out as well as overall shorter first pass, especially when compared with healthy tissues [27-29]. It should be noted that the associations between DW/DCE-MRI parameters and histologic response may not be equated with associations between the same DW/DCE-MRI parameters and EFS (or overall survival). All response or prognostic indicators must be verified independently [30]. In the current study, lower pre- and postKtrans in the tumoral area were significantly associated with better overall survival.

To our knowledge, it is one of the first studies to identify the diagnostic imaging values in peritumoral areas. Although few studies had reported the relationship between the tumoral area MR imaging and EFS or overall survival, we focused the adjacent area to supplement the understanding of MR imaging in predicting prognosis. Meanwhile, parameters in the peritumoral area may be better in consistency compared with the tumoral area that may be influenced by complex circumstances such as necrosis, hemorrhage, edema, and newly formed capillaries. Slaughter et al hypothesized that a residual "alterated field" in the area adjacent to the tumor could be the leading cause of treatment failure and local recurrence [21]. Several studies emphasized on the relationships between molecular marker and therapeutic effect and recurrence [31], and the silencing of cancer-related genes via epigenetic alterations which have been shown to play an important role in tumor progression and to be an early event in the carcinogenesis process [32]. We focused on the diagnostic imaging difference in the peritumoral area, hoping to offer effective suggestions in the clinical treatment of OS. All parameters were independently analyzed and the results showed that lower pre-Ve instead of post-Ktrans was significantly associated with better EFS, and lower pre- and post-Ve were significantly predictive of better overall survival. In Guo et al's study, the tumoral area was defined as outer and inner halves; the results showed that the difference of Ve between the outer and inner halves of tumor at baseline (pre-treatment) was significantly associated with the EFS. Although the definition of parameters was different (they divided the tumoral area as outer and inner halves, and we defined the peritumoral area within $2 \mathrm{~cm}$ around the tumor boundary), the results may have implications that peritumoral pre-Ve (consistent with their Ve at baseline) in our study to some extent is similar with the outer minus inner Ve, and the variation trend may change from peritumoral area to tumor core, which needs further confirmation. Moreover, higher post-ADC in peritumoral area was marginally associated with better overall survival. Recently, one similar study reported that the tumor surface regularity was a predictor of survival for patients who underwent complete resection in glioblastoma patients, indirectly illustrating the significance of peritumoral area [33].

There are some limitations to this study. First is the singlecentered study and limited case number. With the number of patients 34 , two parameters were marginally significant that may need enlarged patient number. Second, peritumoral pathological sequencing should be performed to further demonstrate the correlation between imaging parameters and gene variations. Third, chemotherapy regimen was fixed; results can only prove the relationship between imaging parameters and chemotherapy response in such specified condition.

\section{Conclusion}

In conclusion, we found that tumoral and peritumoral parameters can reflect the chemotherapy response and predict EFS and overall survival, which to some extent supplement and improve the diagnostic and prognostic value of MR imaging in OS patients.

Funding This study has received funding from the National Natural Science Foundation of China (81501434) and Basic Research Program of Natural Science in Shaanxi Province-General Projects (2019JM-264).

\section{Compliance with ethical standards}

Guarantor The scientific guarantor of this publication is Yin Hong, Department of Radiology, Xijing Hospital, Fourth Military Medical University.

Conflict of interest The authors of this manuscript declare no relationships with any companies whose products or services may be related to the subject matter of the article.

Statistics and biometry One of the authors, Li Fan, has significant statistical expertise.We would also like to thank Jialiang Ren from the GE healthcare for his contribution to statistical analysis.

Informed consent Written informed consent was obtained from all subjects (patients) in this study.

Ethical approval Institutional Review Board approval was obtained.

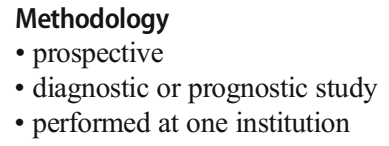

Open Access This article is licensed under a Creative Commons Attribution 4.0 International License, which permits use, sharing, adaptation, distribution and reproduction in any medium or format, as long as you give appropriate credit to the original author(s) and the source, provide a link to the Creative Commons licence, and indicate if changes were made. The images or other third party material in this article are included in the article's Creative Commons licence, unless indicated otherwise in a credit line to the material. If material is not included in the article's 
Creative Commons licence and your intended use is not permitted by statutory regulation or exceeds the permitted use, you will need to obtain permission directly from the copyright holder. To view a copy of this licence, visit http://creativecommons.org/licenses/by/4.0/.

\section{References}

1. Morrow JJ, Bayles I, Funnell APW et al (2018) Positively selected enhancer elements endow osteosarcoma cells with metastatic competence. Nat Med 24:176-185. https://doi.org/10.1038/nm.4475

2. Huvos AG, Rosen G, Marcove RC (1977) Primary osteogenic sarcoma: pathologic aspects in 20 patients after treatment with chemotherapy en bloc resection, and prosthetic bone replacement. Arch Pathol Lab Med 101:14-18

3. Raymond AK, Chawla SP, Carrasco CH et al (1987) Osteosarcoma chemotherapy effect: a prognostic factor. Semin Diagn Pathol 4: 212-236

4. Rosen G, Caparros B, Huvos AG et al (1982) Preoperative chemotherapy for osteogenic sarcoma: selection of postoperative adjuvant chemotherapy based on the response of the primary tumor to preoperative chemotherapy. Cancer 49:1221-1230. https://doi.org/10.1002/10970142(19820315)49:6<1221::AID-CNCR2820490625>3.0.CO;2-E

5. Meyers PA, Schwartz CL, Krailo M et al (2005) Osteosarcoma: a randomized, prospective trial of the addition of ifosfamide and/or muramyl tripeptide to cisplatin, doxorubicin, and high-dose methotrexate. J Clin Oncol 23:2004-2011. https://doi.org/10.1200/JCO.2005.06.031

6. Goorin AM, Schwartzentruber DJ, Devidas M et al (2003) Presurgical chemotherapy compared with immediate surgery and adjuvant chemotherapy for nonmetastatic osteosarcoma: Pediatric Oncology Group Study POG-8651. J Clin Oncol 21:1574-1580. https://doi.org/10.1200/JCO.2003.08.165

7. Saeter G, Hoie J, Stenwig AE, Johansson AK, Hannisdal E, Solheim OP (1995) Systemic relapse of patients with osteogenic sarcoma. Prognostic factors for long term survival. Cancer 75: 1084-1093. https://doi.org/10.1002/1097-0142(19950301)75: 5<1084::aid-cncr2820750506>3.0.co;2-f

8. Enneking WF, Spanier SS, Goodman MA (1980) A system for the surgical staging of musculoskeletal sarcoma. Clin Orthop Relat Res 106-120. https://doi.org/10.1097/00003086-198011000-00013

9. Tofts PS, Brix G, Buckley DL et al (1999) Estimating kinetic parameters from dynamic contrast-enhanced T(1)-weighted MRI of a diffusable tracer: standardized quantities and symbols. J Magn Reson Imaging 10:223-232. https://doi.org/10.1002/(sici)15222586(199909)10:3<223::aid-jmri2>3.0.co;2-s

10. Verstraete KL, Lang P (2000) Bone and soft tissue tumors: the role of contrast agents for MR imaging. Eur J Radiol 34:229-246. https://doi.org/10.1016/s0720-048x(00)00202-3

11. Baur A, Reiser MF (2000) Diffusion-weighted imaging of the musculoskeletal system in humans. Skeletal Radiol 29:555-562. https:// doi.org/10.1007/s002560000243

12. van Rijswijk CS, Kunz P, Hogendoorn PC, Taminiau AH, Doornbos J, Bloem JL (2002) Diffusion-weighted MRI in the characterization of soft-tissue tumors. J Magn Reson Imaging 15:302307. https://doi.org/10.1002/jmri.10061

13. Lang P, Wendland MF, Saeed M et al (1998) Osteogenic sarcoma: noninvasive in vivo assessment of tumor necrosis with diffusionweighted MR imaging. Radiology 206:227-235. https://doi.org/10. 1148/radiology.206.1.9423677

14. Intven M, Reerink O, Philippens ME (2013) Diffusion-weighted MRI in locally advanced rectal cancer: pathological response prediction after neo-adjuvant radiochemotherapy. Strahlenther Onkol 189:117-122. https://doi.org/10.1007/s00066-012-0270-5
15. Intven M, Reerink O, Philippens ME (2015) Dynamic contrast enhanced MR imaging for rectal cancer response assessment after neo-adjuvant chemoradiation. J Magn Reson Imaging 41:16461653. https://doi.org/10.1002/jmri.24718

16. Lambregts DM, Vandecaveye V, Barbaro B et al (2011) Diffusionweighted MRI for selection of complete responders after chemoradiation for locally advanced rectal cancer: a multicenter study. Ann Surg Oncol 18:2224-2231. https://doi.org/10.1245/s10434-011-1607-5

17. Lambrecht M, Vandecaveye V, De Keyzer F et al (2012) Value of diffusion-weighted magnetic resonance imaging for prediction and early assessment of response to neoadjuvant radiochemotherapy in rectal cancer: preliminary results. Int J Radiat Oncol Biol Phys 82: 863-870. https://doi.org/10.1016/j.ijrobp.2010.12.063

18. Kim SH, Lee JY, Lee JM, Han JK, Choi BI (2011) Apparent diffusion coefficient for evaluating tumour response to neoadjuvant chemoradiation therapy for locally advanced rectal cancer. Eur Radiol 21:987-995. https://doi.org/10.1007/s00330-010-1989-y

19. Kim SH, Lee JM, Gupta SN, Han JK, Choi BI (2014) Dynamic contrast-enhanced MRI to evaluate the therapeutic response to neoadjuvant chemoradiation therapy in locally advanced rectal cancer. J Magn Reson Imaging 40:730-737. https://doi.org/10.1002/jmri.24387

20. Hotker AM, Garcia-Aguilar J, Gollub MJ (2014) Multiparametric MRI of rectal cancer in the assessment of response to therapy: a systematic review. Dis Colon Rectum 57:790-799. https://doi.org/ 10.1097/DCR.0000000000000127

21. Slaughter DP, Southwick HW, Smejkal W (1953) Field cancerization in oral stratified squamous epithelium; clinical implications of multicentric origin. Cancer 6:963-968. https://doi.org/10.1002/ 1097-0142(195309)6:5<963::aid-cncr2820060515>3.0.co;2-q

22. Ren L, Mendoza A, Zhu J et al (2015) Characterization of the metastatic phenotype of a panel of established osteosarcoma cells. Oncotarget 6:29469-29481. https://doi.org/10.18632/oncotarget.5177

23. Hayashida Y, Yakushiji T, Awai K et al (2006) Monitoring therapeutic responses of primary bone tumors by diffusion-weighted image: initial results. Eur Radiol 16:2637-2643. https://doi.org/ 10.1007/s00330-006-0342-y

24. Guo J, Glass JO, McCarville MB et al (2015) Assessing vascular effects of adding bevacizumab to neoadjuvant chemotherapy in osteosarcoma using DCE-MRI. Br J Cancer 113:1282-1288. https://doi.org/10.1038/bjc.2015.351

25. Parker GJ (2004) Analysis of MR diffusion weighted images. Br J Radiol 77 Spec No 2:S176-S185. https://doi.org/10.1259/bjr/ 81090732

26. Reddick WE, Wang S, Xiong X et al (2001) Dynamic magnetic resonance imaging of regional contrast access as an additional prognostic factor in pediatric osteosarcoma. Cancer 91:2230-2237. https://doi.org/10.1002/1097-0142(20010615)91:12<2230::aidcncr1253>3.0.co;2-t

27. Maas M, Nelemans PJ, Valentini V et al (2010) Long-term outcome in patients with a pathological complete response after chemoradiation for rectal cancer: a pooled analysis of individual patient data. Lancet Oncol 11:835-844. https://doi.org/10.1016/S1470-2045(10)70172-8

28. Beets-Tan RG, Beets GL (2004) Rectal cancer: review with emphasis on MR imaging. Radiology 232:335-346. https://doi.org/10. 1148/radiol.2322021326

29. Leach MO, Brindle KM, Evelhoch JL et al (2005) The assessment of antiangiogenic and antivascular therapies in early-stage clinical trials using magnetic resonance imaging: issues and recommendations. Br J Cancer 92:1599-1610. https://doi.org/10.1038/sj.bjc.6602550

30. Hawkins DS, Rajendran JG, Conrad EU 3rd, Bruckner JD, Eary JF (2002) Evaluation of chemotherapy response in pediatric bone sarcomas by [F-18]-fluorodeoxy-D-glucose positron emission tomography. Cancer 94:3277-3284. https://doi.org/10.1002/cncr.10599

31. van Houten VM, Leemans CR, Kummer JA et al (2004) Molecular diagnosis of surgical margins and local recurrence in head and neck 
cancer patients: a prospective study. Clin Cancer Res 10:3614 3620. https://doi.org/10.1158/1078-0432.CCR-03-0631

32. Martone T, Gillio-Tos A, De Marco L et al (2007) Association between hypermethylated tumor and paired surgical margins in head and neck squamous cell carcinomas. Clin Cancer Res 13: 5089-5094. https://doi.org/10.1158/1078-0432.CCR-07-0119

33. Perez-Beteta J, Molina-Garcia D, Ortiz-Alhambra JA et al (2018) Tumor surface regularity at MR imaging predicts survival and response to surgery in patients with glioblastoma. Radiology 288: 218-225. https://doi.org/10.1148/radiol.2018171051

Publisher's note Springer Nature remains neutral with regard to jurisdictional claims in published maps and institutional affiliations. 